\title{
Aproximación teórica a la relación entre los términos gestión documental, gestión de información y gestión del conocimiento
}

\author{
Verónica Gauchi Risso*
}

Resumen: El objeto de esta exposición consiste en el abordaje teórico de los términos gestión documental, gestión de información y gestión del conocimiento y las relaciones existentes entre ellos. Este trabajo corresponde a un estudio de revisión o compilación de tipo descriptivo, en el que los términos seleccionados son descritos uno a uno en sus características y que tiene por finalidad transmitir informaciones válidas científicamente con el objeto de establecer una relación entre los términos propuestos. Tres aspectos confluyen en la conceptualización: documentos, tecnologías de la comunicación y personas; la combinación de todas ellas da lugar a una serie de capacidades que son lo que constituye el capital de toda organización; este proceso evidencia una relación estrecha entre la gestión documental, de información y del conocimiento que conforman un equipo complementario e imprescindible en toda organización.

Palabras clave: Gestión documental, gestión de información, gestión del conocimiento.

\section{Theoretical approach to the relation among the terms «record management», «information management» and «knowledge management»}

Abstract: The purpose of this paper is to offer a theoretical approach to the terms urecord management", "information management" and "knowledge management", as well as to the relationships among them. This work consists of a descriptive review of the pertinent literature in which the chosen terms are described individually. It aims to transmit scientifically valid information in order to establish a relationship among the three. Three aspects are drawn together in the conceptualization: documents, communication technologies, and people, the combination of which gives rise to a number of strengths that constitute the capital of any organization. This process provides evidence of a strong relationship among record management, information management and knowledge

* Universidad Nacional de Mar del Plata, Fac. Humanidades, Argentina.

* Universidad de Granada, Fac. Comunicación y Documentación, España, Correo-e: vebega@ mdp.edu.ar, vebega@correo.ugr.es

Recibido: 07-06-2011, 2. ${ }^{\text { }}$ versión, 09-01-2012, aceptado: 03-05-2012 
management, which together represent a complementary and indispensable grouping in any organization.

Keywords: Record management, information management, knowledge management.

\section{Introducción}

Desde 1970 la irrupción de una nueva gama de tecnologías destinadas a manipular y transmitir información ha creado un panorama completamente diferente en cuanto a que las personas han incorporado nuevas capacidades como una extensión de su naturaleza, hasta el punto de convertirlas en imprescindibles para vivir en el mundo actual; en consecuencia, aquello que imprecisamente se mencionaba como "revolución informática" ha evolucionado en "revolución tecnológica»; y no se trata de una cuestión semántica menor, sino de la diversidad y desarrollo que sobrelleva el progreso en este ámbito. Sin embargo la variación más significativa se coloca a principios de 1990, con el impulso y la generalización del uso de las redes de comunicación, puesto que ya no se trata únicamente de gestionar información, sino de transmitirla, lo cual ha asentado los inicios de la Sociedad de la Información y, mucho antes que ésta haya sido realmente asumida y consolidada, surge la Sociedad del Conocimiento.

Las necesidades derivadas de la incorporación de las Tecnologías de la Información y la Comunicación (TIC) a la vida cotidiana van cambiando y obligan continuamente a ofrecer soluciones a la gestión, transacción e interacción de la información. Actualmente escuchamos hablar de gestión documental, gestión de información y gestión del conocimiento, términos todos ligados a las variaciones que ha sobrellevado el desarrollo tecnológico en nuestro campo de trabajo y que han proyectado nuevos perfiles profesionales; sin embargo, posiblemente aún no se ha llegado a establecer de forma precisa cuál es el verdadero calado de su significado y cuáles van a ser los efectos reales en las formas de vivir, de trabajar y de producir para las próximas décadas. El desarrollo de las TIC, el fenómeno de la explosión de la información y la variedad de formas en que es posible compartirla han generado nuevos desafíos: a medida que avanza la tecnología, la estabilidad y la perdurabilidad de los documentos disminuyen considerablemente; se multiplican las demandas de soluciones concretas para agilizar el proceso documental; se automatizan los flujos de trabajo; se ofrecen servicios por vía telemática; las organizaciones necesitan coordinar y controlar de forma sistemática la creación, recepción, organización, almacenamiento, preservación, acceso, difusión y eliminación de sus documentos y de la información que generan y reciben.

Hoy en día se dispone de más información de la que podemos procesar y asimilar, y es precisamente esta sobreabundancia de información o infoxicación ${ }^{1}$ (Cornella, 2009) el principal problema para su gestión; por lo que las organizaciones que se desenvuelven en este nuevo entorno deben ser capaces de diferenciar la información mas relevante y de seleccionar aquélla que resulte pertinente; en definitiva, en estos momentos la información adquiere un rol fundamental 
Aproximación teórica a la relación entre los términos gestión documental, gestión de información...

dentro de las organizaciones, y por ello un valor inigualable, puesto que se ha convertido en el factor crítico que posibilita que las organizaciones reaccionen frente al cambio externo constante, con renovación e innovación constantes.

\section{Material y métodos}

El objetivo de esta exposición consiste en el abordaje teórico de los términos ${ }^{2}$ gestión documental, gestión de información y gestión del conocimiento. Indudablemente en su evolución nuestra disciplina ha incorporado herramientas, técnicas, métodos y nuevos términos, lo cual nos lleva a inspeccionar si su progreso y fortalecimiento como un campo del conocimiento se encuentra vinculado de forma directa a la consolidación de estos conceptos y si existe un corpus teórico identificable y consensuado por la comunidad científica en torno a ellos. Esta tarea podría parecer una cuestión de poca importancia, sin embargo, y debido a la cantidad, variedad y calidad de información expuesta en torno a ellos, la falta de acuerdo respecto de su delimitación, y la observación de una marcada ambigüedad y confusión sobre su significado y demarcación, consideramos necesario formalizar una caracterización de los términos y de las relaciones ${ }^{3}$ que concurren entre ellos. Tras efectuar una revisión de lo publicado durante los últimos años en torno a estas cuestiones resulta indudable que el lector tendrá que enfrentarse a una semántica confusa y difusa, a la vez que encarar diferentes versiones conceptuales del mismo término. En virtud de este contexto, este trabajo corresponde a un estudio de revisión o compilación de tipo descriptivo, en el que los términos seleccionados son representados uno a uno en sus características; y que tiene por finalidad transmitir informaciones válidas científicamente con el objeto de establecer una concordancia entre los términos propuestos.

\section{Marco conceptual}

\subsection{Acerca del término gestión}

Preliminarmente al desarrollo de cualquier tipo de formalización teórica resulta imprescindible definir el término gestión, puesto que todos los conceptos implicados en este trabajo hacen referencia a la gestión en sus diferentes prácticas y/o ámbitos de aplicación. El diccionario de la RAE define gestión $n^{4}$ como el: "conjunto de trámites que se llevan a cabo para resolver un asunto"; como segunda opción, refiere a la: "dirección, administración de una empresa, negocio, etc." Ambas definiciones hacen referencia a la acción y al efecto de administrar.

A la luz de la definición, el término gestión parecería encontrarse equiparado al de administración, sin embargo y tal como se concibe actualmente la gestión dentro de la perspectiva de las prácticas organizativas, ésta comporta un conjunto de actividades de decisión que tienen lugar dentro de una organización y es aplicada como un conjunto de procedimientos de adecuación de recursos de 
cualquier índole a aquellos fines para los cuales han sido obtenidos los recursos; por tanto, gestionar consiste en seleccionar ciertas acciones partiendo de diversas informaciones, y compone o dibuja un conjunto de actividades entrelazadas que incluyen, al menos, a la administración de los recursos, el número de actos programados y una línea de trabajo abordada, a corto, medio y largo plazo; todas ellas circunscriben y comprenden al concepto administración. Para los teóricos de la administración, ésta se contrapone a gestión, puesto que la administración hace referencia a la capacidad de representar a la empresa ante terceros, en cambio gestión alude al ámbito interno de la empresa.

Por tanto, gestionar es hacer que las decisiones se ejecuten, tramitar asuntos con vistas a la obtención de unos resultados, involucra el conjunto de proposiciones teóricas que explican el uso de unas reglas, procedimientos y modos operativos para llevar a cabo con eficacia las actividades económicas que permiten lograr los objetivos de una organización. Con relación a la dirección, la gestión, es mucho más restringida, puesto que la dirección abarca un proceso más general por el cual se conduce a una organización hacia sus objetivos, mediante la aplicación de los factores disponibles, y desarrollando las funciones de: planificación, organización, gestión y control; principios directivos que sirven para toda clase de instituciones.

\subsection{Singularidades del término gestión documental}

A partir de 1950 la gestión documental (GD) ha sido un específico ámbito de trabajo de la archivística (Cruz Mundet, 2006), los archiveros evolucionaron paulatinamente hacia la idea de gestor de documentos (records management) y la profesión comenzó a ser concebida, especialmente por la corriente norteamericana, como gestión documental. A este respecto Elio Lodolini (1993) señala que "durante la década de los cincuenta comenzaron las primeras acciones de carácter práctico y luego se desarrolló en la literatura norteamericana el concepto de Record Management». Estas actividades promovidas al término de la Segunda Guerra Mundial, ya que resultaba imperioso organizar la información acumulada por el gobierno estadounidense tras la guerra, fueron utilizadas para mejorar los aspectos administrativos en la generación de la documentación pública. "Empezó entonces la colaboración entre los Archivos Federales y las oficinas administrativas: se crearon los "records officers" y se formaron comisiones para estudiar conjuntamente los problemas" (Llansó, 1993); hacia finales de los años cuarenta nos encontramos con la figura del records manager o gestor de documentos y la aceptación del término records management, o Gestión de Documentos, término que ya era utilizado en las empresas privadas, y que luego en 1950 cuando se crea la Federal Records Act, se define por primera vez desde el punto de vista legal, incluyendo la creación y conservación de los documentos, su destrucción y transferencia.

En ese momento algunos archiveros norteamericanos (William Benedon, Jessie L. Clark, Theodore R. Schellenberg, Emmet J. Leahy, Wilmer O. Maedke, Mary 
F. Robek y Gerald Brown) arribaron a la idea de que la gestión de la documentación administrativa debía comenzar desde el mismo momento en que se creaba el documento y debía abarcar todo su ciclo vital ${ }^{5}$, hasta su transferencia a los archivos históricos, los documentos de valor primario ${ }^{6}$ deberían ser evaluados y seleccionados por sus creadores, con la ayuda de los archiveros. En este sentido Schellemberg (1956) distinguía entre los profesionales que trabajaban con los documentos de uso inmediato e intermedio, identificándolos como gestores documentales, y los profesionales que trabajaban con los documentos de valor secundario $^{7}$ o histórico, reconociéndolos como archiveros. El mismo problema ha llevado a algunos colegas franceses, después de un debate terminológico con otros países francófonos, a proponer el uso de "gestion des informations et des documents d'activités para referirse a este campo (Bustelo Ruesta, 2011a).

La transformación radica en que la archivística dividió el ciclo de vida del documento en tres edades ${ }^{8}$ : activa, semiactiva e inactiva, concibiendo que los documentos con vigencia administrativa y con un alto grado de uso se incluyen dentro de la primera etapa y se conservan en los archivos de gestión o de oficina; los que continúan vigentes pero resultan de poca consulta se encuentran en una fase semiactiva y se transfieren a un archivo general; finalmente, aquellos documentos que han perdido su vigencia administrativa pasan a la fase inactiva y tienen dos posibles destinos: el archivo histórico para los documentos con valores permanentes y la destrucción para aquellos que no poseen ninguna valía. La diferencia radica en que la archivística clásica, desarrollada durante el siglo XIX, restringió el papel de los archiveros a la última de las etapas: los archiveros eran únicamente los custodios de la documentación histórica estando, esencialmente, al servicio de los historiadores. Esta concepción estática de la archivística dominó la teoría y la práctica profesional durante el siglo XIX, pero a principios del Xx saltó en pedazos con la irrupción de las masas documentales generadas a partir de la Primera Guerra Mundial; y como es sabido, son las necesidades nacidas de la crisis económica de 1930 y sobre todo la Segunda Guerra Mundial las que forzaron a los gobiernos y a los archiveros a abordar formalmente los problemas planteados por el aumento incontrolado de la masa documental.

En España el término GD fue introducido a finales de la década de 1980 y principios de la siguiente, en virtud de los contactos establecidos entre la Asociación de Archiveros Catalanes y Michel Roberge (2006)9; quien la define como "el conjunto de operaciones y técnicas relativas a la concepción, al desarrollo, a la implantación y a la evaluación de los sistemas administrativos necesarios, desde la creación de los documentos hasta su destrucción o su transferencia a los archivos"; sin embargo la GD actualmente comprende algo más que una readecuación y renovación de la tarea de los archiveros, puede considerarse como un proceso vital para toda organización debido a la magnitud que alcanza la producción de documentos, que permiten analizar y controlar sistemáticamente cómo la información registrada se crea, recibe, mantiene o utiliza.

Como ya mencionamos con «el desarrollo de las TIC cambia la organización del trabajo en las oficinas, se plantean asi nuevos problemas que exigen la apli- 
cación de nuevas técnicas para su tratamiento archivístico y un fortalecimiento de las interrelaciones existentes entre las fases del ciclo de vida de los documentos" (Llansó, 1993); y es en este contexto, y al amparo de la archivística cuando la gestión documental hizo su aparición. Como consecuencia, el término documento $^{10}$ se especifica como: «información creada o recibida, conservada como información y prueba, por una organización o un individuo en el desarrollo de sus actividades o en virtud de sus obligaciones legales" (Norma ISO UNE 15489, 2006). Así la GD no sólo interviene sobre la documentación generada sino además hacia el interior de la organización en sus propios ciclos documentales, comenzando en el nivel primario de la producción y en las relaciones que se deben producir entre las diferentes áreas y actividades de trabajo que implican de alguna forma la generación de documentos. La tarea no sólo reside en las operaciones producidas sobre la documentación, sino en definir cómo se genera y de dónde emana la documentación, lo cual implica el rediseño de métodos y circuitos de trabajo y el establecimiento de procedimientos; todo ello bajo una metodología de normalización, racionalidad y economía de recursos.

Una característica de la GD es su transversalidad en toda una organización, poniendo énfasis en la vinculación con todas las actividades y otorgando una alta prioridad al valor primario del documento, es decir, a la utilidad de la documentación para la acción del quehacer organizacional; sin lugar a dudas esta concepción varió sensiblemente el desempeño del archivero ampliando sus tareas desde el momento en que se da origen al documento. Si bien a menudo se asocia la GD con la implementación de un programa informático, no necesariamente debe ser así, en algunas ocasiones una solución de GD puede pasar por fijar unas pautas corporativas; las TIC por sí mismas no constituyen un factor de éxito, aunque pueden contribuir de manera significativa a conseguirlo. La integración de soluciones tecnológicas puede ser un elemento imprescindible, porque ya no se puede depender exclusivamente de las transferencias físicas o de las hojas de remisión para el control de la documentación, en la mayoría de los casos resulta necesaria la implantación de un sistema que ofrezca los datos necesarios sobre los documentos, desde que nacen, hasta que se guardan permanentemente o destruyen.

Los Sistemas de Gestión Documental (SGD) ${ }^{11}$ agrupan operaciones y técnicas de la gestión administrativa general con el objeto de coordinar y controlar todas aquellas funciones y actividades específicas que afectan la creación, recepción, ubicación, acceso y preservación de los documentos, protegiendo sus características estructurales y contextuales con el objeto de garantizar su autenticidad e integridad a lo largo del tiempo; la integración de los procesos y controles documentales en los procesos de trabajo debe ser el objetivo principal de cualquier modelo de gestión de documentos, porque permite: la disminución del tiempo de localización y recuperación de los documentos ${ }^{12}$, la disminución, de espacio físico de almacenamiento, aumenta la seguridad mediante una política de back-up correcta y acrecienta la rapidez en la atención al cliente, incluso actualmente existen soluciones opensource ${ }^{13}$ que brindan funcionalidades similares a las soluciones comerciales. 
FIGURA 1

Sistema de gestión documental

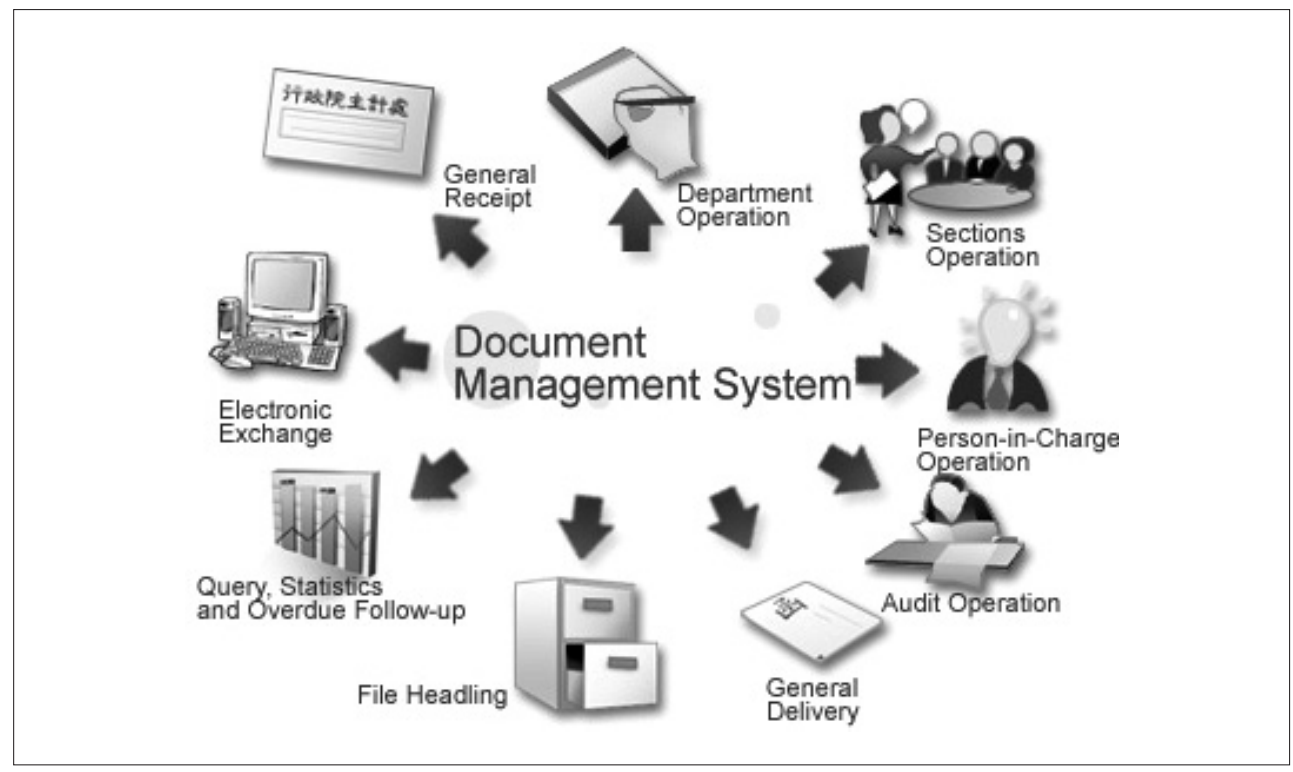

Fuente: Disponible en: <http://www.gestion.org/articulos/documental/sistemas-de-gestion-de-contenidos>.i

A partir de las quiebras de Enron ${ }^{14}$, A. Andersen y Worldcom, y la ocultación y destrucción de información comprometida, la GD se colocó en una posición estratégica dentro de las organizaciones, sobre todo por la necesidad de operar de manera efectiva la documentación, no sólo aquella que se encontraba en papel, sino la que se genera por sistemas electrónicos; ciertamente a partir de estos sucesos, se comprueba un progresivo interés de muchas organizaciones por asegurar las pruebas de sus actividades a través de los documentos (Alòs Moner, 2011).

La normalización de sistemas de GD se ha regulado con la Norma ISO 154891:2001 ${ }^{15}$ : "Información y documentación-Gestión de documentos", continuadora del modelo de las Normas 9000, 14000, 27000 y algunas más no tan conocidas; la norma plantea que los gestores de documentos e información tienen el cometido de poner en valor los recursos informativos disponibles, para documentar los procesos que se están llevando a cabo en la organización en un momento dado. El éxito en la adopción de esta norma favoreció la concreción de una nueva propuesta de reconocimiento de un sistema de gestión para los documentos (Bustelo Ruesta, 2011b) que bajo el título "Management system for records" (MSR) conformó como serie ISO 30300 al sistema normalizado de gestión documental. Las ISO 30300:2011 «Management Systems for Records - Fundamentals and vocabulary» e ISO 30301 "Management systems for records - Requirements Adapting”; que si bien se basan en la Norma ISO 15489 tienen un enfoque dife- 
rente, la gran diferencia radica en el hecho que la 30300 es una norma de rango Management System Standard (MSS) que interviene en los requisitos que debe cumplir cualquier sistema organizacional para garantizar un funcionamiento orientado a la mejora continua, por la que cualquier sistema integrado de gestión debe ser la suma de: calidad + medioambiente + seguridad de la información + prevención de riesgos laborales + gestión documental; esta norma puede considerarse como una guía para la gestión de documentos de archivo de una organización, independientemente del soporte en el que se encuentren. "La serie ISO 30300, aceptada por ISO como modelo de gestión estandarizado, se muestra como lo que es: un abanico interminable de oportunidades para el archivero creativo, dotado de la capacidad de apreciar la innovación en un entorno orientado hacia la mejora continuada" (Moro Cabero, 2011).

\subsection{Singularidades del término gestión de información}

Las investigaciones realizadas en torno al término información se dividen básicamente entre las que la consideraron como algo externo, objetivo, tangible, y las que la contemplaron como algo subjetivo, cognitivo y situacional. Entre los años 1928 y 1948, la Teoría matemática de la comunicación ${ }^{16}$ propuesta por Claude Shannon (1949), definió el término información teniendo como referencia proporciones o cantidades representadas por una función logarítmica en base 2, por la cual la cantidad de información contenida en un mensaje es un valor matemático bien definido y medible. La información era tratada cuantitativamente y sólo se ocupaba de sus elementos sintácticos obviando por completo los aspectos relacionados con la semántica, únicamente se refería a las condiciones técnicas que permitían la transmisión de mensajes, lo cual no decía nada acerca de la información en sí misma, sino a la posibilidad de que un mensaje, dentro de un conjunto de mensajes posibles, sea recibido.

A finales de la década de 1970 la información fue definida en términos semánticos y cognitivos; bajo esta nueva concepción se prestó atención al contexto social y a la interacción entre grupos, individuos y sistemas, la información se aprecia desde posiciones interpretativas e históricas, donde la percepción se encuentra afectada por factores culturales. Uno de los primeros en aceptar la idea e insistir en la importancia del enfoque cognitivo en ciencia de la información fue Bertram Brookes (1980) quien indica que la información es una entidad presente en toda la actividad humana, por lo que cada persona tiene una imagen mental diferente de su entorno físico que establece una relación simple entre nuestras imágenes mentales y las realidades físicas que observamos. Bajo esta nueva visión, la información fue pensada como «una colección de signos estructurados con un fin determinado por un remitente; con la intención de cambiar la estructura de las imágenes de un receptor; es la información entonces, [...] la estructura de todo texto capaz de cambiar la estructura de las imágenes de algún recepton (Belkin, 1978). Este enfoque inserta a la información en el ámbito de los procesos de comunicación social, incorpora los conceptos comunicación y 
conocimiento, que desde el prisma de lo cognitivo se vuelven imprescindibles en la comprensión del fenómeno, y niega explícitamente que la información sea una cosa, sino que muy por el contrario se ve como un cambio en el conocimiento del receptor; la información ${ }^{17}$ es identificada con el contenido semántico de los datos. Habiendo repasado cuáles son los dos grandes matices teóricos que han teñido la definición de información, repasemos ahora el desarrollo que ha tenido el término gestión de información.

La primera vez que aparece la utilización del término Gestión de Información (GI - Information Management) fue en una conferencia titulada "Information Management in Engineering Education» presentada por Ellis Mount (1966) en el marco de la Conferencia sobre fuentes de información, sistemas y medios de comunicación en la formación de Ingenieros, llevada a cabo en la Universidad de Lehigh (EE.UU.) en Mayo de 1920; el término fue aplicado a una subdisciplina de la ingeniería enfocada a ofrecer a los estudiantes una teoría y uso de la información como complemento de su aprendizaje y de la práctica de su especialidad. Sin embargo el nacimiento concreto del término comienza, con los primeros trabajos del Congreso Estadounidense sobre la reducción de trámites burocráticos, que culminó con la aprobación de la Ley denominada "Paperwork Reduction Act» que llegó en 1980 luego de una década de debates sobre el "gravamen del papel»; si bien esta legislación no establece cuestión alguna acerca de la gestión del recurso de la información, dispone el nombramiento por parte de las agencias federales de un responsable para llevar a cabo en su ámbito la política de información federal en todas las actividades relacionadas con la recogida, uso y diseminación de la información.

Sobre esta cuestión Martin White, miembro del Institute of Information Scientists, escribía: "Hay una tendencia lamentable a definir el nacimiento de la gestión de la información el día en que el Acta de Reducción de trámites burocráticos entró en el código de leyes de los EE.UU., de América. En realidad, la gestión de la información tiene por lo menos dos siglos de existencia y se ha enmascarado bajo el nombre de "inteligencia militar"” (White, 1985); el editor del libro en el que aparece este estudio, Blaise Cronin, expresa que ésta es la definición más interesante de la nueva disciplina; sin duda esta definición revela un aspecto esencial, olvidado durante siglos, la relación evidente entre información y eficaciaéxito, esencialmente en el campo donde mejor se formula la competitividad y la lucha por la supervivencia: la guerra (López Hernández, 1990). GI se produjeron principalmente de la mano de Blaise Cronin; en 1979 el Institute of Information Scientists convocó en Londres a una conferencia con el título Gestión efectiva de la información; esta nueva disciplina influyó en el cambio de denominación de la Association of Special Libraries and Information Bureaux (ASLIB), que después de 50 años, fue modificada por el de Association for Information Management; entidad que desde 1981 edita la revista especializada International Journal of Information Management.

Pero fue el sociólogo japonés Yoneji Masuda, quien en su libro La Sociedad de la Información como Sociedad Postindustrial (editado en 1980) desarrolló la 
concepción actual de sociedad de la información. A partir de un informe del Ministerio de Industria y Comercio de su país, Masuda elaboró para el Instituto JACUDI el llamado "Plan para la Sociedad de la Información; un objetivo nacional para el año 2000", conocido como el Plan JACUDI; en su concepción la sociedad de la información es una: "sociedad que crece y se desarrolla alrededor de la información y aporta un florecimiento general de la creatividad intelectual bumana, en lugar de un aumento del consumo material" (Masuda, 1984). El cambio central en el funcionamiento de la sociedad iniciado en la década de 1970 supuso un traslado de los medios de generación de riqueza del área industrial a la de servicios, especialmente en la generación, almacenamiento, procesamiento y distribución de todo tipo de información donde desempeñan un papel principal las TIC, no obstante, la información no se almacena con facilidad en las computadoras, y no está constituida por datos, cuanto mas complejo sea el modelo de la información, menos útil será porque la información tiene múltiples significados en una organización, y la tecnología es solo uno de los componentes del entorno de la información y, a menudo, no es la manera mas conveniente de generar el cambio.

A raíz de estas conceptualizaciones durante la década de 1980, empresas y organizaciones reafirmaron la importancia de la información, por lo que en 1990 se originan las grandes inversiones en materia de sistemas informáticos y comienza a hablarse de GI en el ámbito organizacional, de lo que se desprende que la GI surge como una consecuencia lógica de un nuevo tipo de sociedad, la postindustrial, postmoderna o de la información, que ha roto el equilibrio de los tradicionales sectores económicos y que considera la actividad de la información como fuente de poder, detentadora del conocimiento y, en último término, de la capacidad de decisión acertada.

A la hora de caracterizar el término GI se puede establecer una distinción entre disciplina y actividad; Elizabeth Adams la define como disciplina y expresa que la GI es la "función de alta dirección para desarrollar una serie de políticas, programas y procedimientos para planificar, gestionar y controlar eficazmente las necesidades de información y los recursos de soporte de manejo de la información" (White, 1985). Por otro lado Lynda Woodman (1985) que la define como actividad, plantea que "gestión de la información es todo lo que se refiere a conseguir la información adecuada, en la forma adecuada, para la persona adecuada, al coste adecuado, en el momento adecuado, en el lugar adecuado, para tomar la acción adecuada". Para representar una progresión más amplia en la secuencia que han sobrellevado las definiciones del término aportaremos la concebida por Gloria Ponjuan Dante (2004), mucho más contemporánea, para la autora la GI hace referencia al "proceso mediante el cual se obtienen, despliegan o utilizan recursos básicos (económicos, físicos, bumanos, materiales) para manejar información dentro y para la sociedad a la que sirve. Tiene como elemento básico la gestión del ciclo de vida de este recurso y ocurre en cualquier organización. Es propia también de unidades especializadas que manejan este recurso en forma intensiva, llamadas unidades de información". 
El objetivo fundamental de la GI consiste en aprovechar la información de los recursos y capacidades de información de la organización con el fin de permitir a la organización adaptarse a su entorno cambiante. Conceptualmente, la GI puede ser pensada como un conjunto de procesos que apoyan y son simétricos a las actividades de aprendizaje de la organización, estos procesos son: la identificación de las necesidades de información, la adquisición de información, la organización y almacenamiento de la información, el desarrollo de productos y servicios de información, la difusión de información, y el uso de la información (Choo, 1995); en el manejo de GI es necesario contar con políticas que sitúen y precisen los espacios, responsabilidades, alcance y otros elementos regulatorios indispensables para una normalización de las actividades; en palabras de Choo, el modelo de GI basado en procesos, debe transitar toda la cadena de valor, comenzar con la identificación de necesidades de información, continuar con la adquisición, organización, almacenamiento, elaboración de productos y prestación de servicios, y distribución, para concluir el ciclo con el uso de la información.

FIGURA 2

\section{Ciclo de la gestión de información}

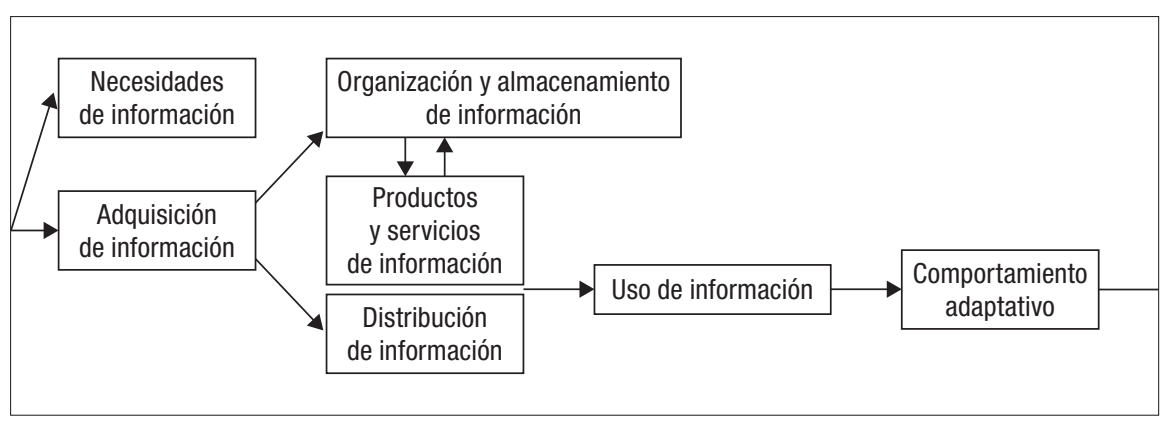

Fuente: Choo, C. W. (1995).

Para Davenport y Prusak (2001) la GI se delimita en un ciclo de actividades informativas relacionadas y sujetas a planificación, diseño y coordinación, que proporciona una perspectiva asentada en procesos y que complementa las perspectivas más tradicionales de la gestión de información como gestión de tecnología o de recursos de información.

Es conveniente indicar que la utilización del término "gestión de contenidos" muchas veces es confundido con el término GI, que se utiliza para englobar toda una serie de tareas relacionadas con la gestión de la información en las empresas; este término que comenzó a ser utilizado en el año 2001, fue rápidamente aceptado por los vendedores de herramientas de software, ávidos de nuevas estrategias comerciales que les permitiesen elevar sus ventas, por lo que en muchas oportunidades ha sido aceptado como denominación de una nueva forma de 
entender la GI en las empresas (en inglés las siglas ECM - Enterprise Content Management), esta concepción entiende la gestión de todo tipo de contenidos creados y/o utilizados en la empresa y su acceso por cualquier persona de la organización.

\subsection{Singularidades del término gestión del conocimiento}

Desde que Bell $(1973 ; 2001)$ definiera la sociedad del conocimiento han ido brotando y atesorando gran renombre un conjunto de expresiones que configuran sus verdaderos contenido y alcance dentro de la comunidad científica; entre otras expresiones, es el caso de: capital humano, capital intelectual, activos intangibles, proceso de aprendizaje, organización inteligente, aprendizaje organizativo, conocimiento explícito, conocimiento tácito, economía del conocimiento y gestión del conocimiento. La Gestión del Conocimiento (GC) es una disciplina que tiene poca historia, y que junto al surgimiento de la sociedad del conocimiento lo que se gestiona es lo intangible, y este nuevo enfoque es el que ha cambiado drásticamente el contenido de lo gestionado.

En 1962 Fritz Machlup, economista y académico austríaco, usó la denominación sociedad del conocimiento en su libro Producción y distribución del conocimiento en los Estados Unidos; según este autor el número de empleos basados en todo el proceso de generación y distribución de información, ya en la década de 1960, era mayor al relacionado con cualquier otro tipo de esfuerzo físico. Según el cálculo de Machlup, ese año, el 35\% del producto interior bruto (PBI) norteamericano procedía del sector de la información, valor que proyectaba hacia el futuro una clara tendencia a convertirse en un descriptor dominante de la actividad productiva.

A fines de la década de 1960, el término fue nuevamente utilizado, en esta ocasión por Peter Drucker, en su libro La era de la discontinuidad (1969); allí fue empleado evocando una etapa posterior a la era de la información, a la que se llegará por medio de las oportunidades que representan los medios y la humanización de las sociedades actuales. Drucker dedicó un capítulo a la "Sociedad del Conocimiento", basándose en datos y proyecciones económicas establecidas en 1962 por Fritz Machlup, a los que Drucker añadió que, a finales de la década de 1970, el sector del conocimiento generaría la mitad del PBI. Drucker invirtió la máxima de que «las cosas más útiles, como el conocimiento, no tienen valor de cambio" erigiendo la importancia del saber como factor económico de primer orden, es decir, introdujo el conocimiento en la ecuación económica y lo mercantilizó; además dejó en claro que lo relevante desde el punto de vista económico no era su cantidad o calidad sino su capacidad para generar riqueza, su productividad. Sin duda, se trataba de un uso particular de la palabra conocimiento, aunque totalmente apropiado al contexto especializado de la teoría económica donde surgen tanto el concepto de Sociedad del Conocimiento como el de Sociedad de la Información. 
Posteriormente en el libro "La sociedad post-capitalista», Drucker (1994) destacaba la necesidad de generar una teoría económica que pusiera al conocimiento en el centro de la producción de riqueza, también aseguraba que en esa inminente sociedad postcapitalista el recurso básico sería el saber, mientras que los tradicionales factores de producción (recursos naturales, mano de obra y capital) serían secundarios y podrían obtenerse, con facilidad, en tanto hubiera saber. En este sentido Drucker pronosticó la emergencia de una nueva capa social de trabajadores del conocimiento y la tendencia hacia una sociedad del conocimiento; sociedad caracterizada por una estructura económica y social en la que el conocimiento sustituye al trabajo, a las materias primas y al capital como la fuente más importante de la productividad, crecimiento y desigualdades sociales.

En la década de 1990 este término fue profundizado en estudios publicados por investigadores como Nico Stehr (1984) y Robin Mansell (1998), cuyos trabajos puntualizan que el acceso a la información, aun si fuera libre y equitativo, no garantiza la incorporación de conocimientos; la información puede ser un instrumento de conocimiento pero no es el conocimiento en sí mismo, porque éste requiere de elementos que pueden ser incorporados como saber, mientras que la información contiene mayoritariamente contenidos determinados por intereses comerciales, que incluso pueden retrasar o interferir con la incorporación de conocimientos.

El término sociedad del conocimiento, preferido por los medios académicos, ha cosechado su mayor impulso cuando la UNESCO (2005) acogió el término "sociedad del conocimiento" o su variante "sociedades del saber" dentro de sus políticas institucionales, desplegando en torno al tema una preocupación al pretender incorporar una idea más integral y no amarrada exclusivamente a la extensión económica. Este concepto más integral incluye dimensiones de transformación social, cultural, económica, política e institucional y una perspectiva más pluralista y asociada al desarrollo sostenible; la GC trata sobre aspectos críticos de la adaptación, sobrevivencia y competencia organizacional para enfrentar el constante y discontinuo cambio ambiental. Esencialmente, implica los procesos organizacionales que buscan una "combinación sinérgica de los datos, información, sistemas de información, y la capacidad creativa e innovadora de seres bumanos" (Malhotra, 2000).

Pero a la luz de esta exposición sobre en qué bases se sustenta la diferenciación entre los términos sociedad de la información y sociedad del conocimiento, porque para ambos las transformaciones sociales han sido las mismas. En principio debemos considerar qué se entiende por información y qué por conocimiento, teniendo en cuenta que: una colección de datos no es información, una colección de información no es conocimiento, una colección de conocimiento no es sabiduría y una colección de sabiduría no es necesariamente la verdad, lo cual establece una diferencia sustancialmente cognitiva. Del mismo modo, la concepción bastante extendida de que la tecnología resolvería todos los problemas de gestión de información ha resultado ser equívoca, en tanto que, si bien en una primera instancia ha ayudado en ese proceso y ha genera- 
do grandes beneficios en las organizaciones, poco a poco se ha ido reproduciendo el problema cotidiano de ignorancia de la localización de los recursos disponibles y, lo que resulta aún peor, el desconocimiento de su propia existencia. Lo que hace rentable a las TIC en una organización no es el mero hecho de tenerlas, sino de cómo se utilizan, de cuan inteligentemente se gestionan; la clave reside en que los sistemas de información nos permitan intercambiar información: transaccionarla. La tendencia a acuñar el término GC responde a la necesidad que vienen planteando las organizaciones de disponer de sistemas de gestión del conocimiento plenamente operativos con la idea de economizar esfuerzos tanto humanos como materiales y de intentar aprovechar las sinergias positivas derivadas de una gestión transaccional de la información en la búsqueda de esas nociones fundamentales que aporten conocimiento sobre determinados temas a los gestores de las mismas (Martínez Méndez, 1995), pero el conocimiento es información con mayor valor y, en consecuencia, representa la forma mas difícil de manejar.

Los últimos años centraron su atención en la GC, en la identificación y gestión de los activos cognitivos (intangibles) de las organizaciones; el conocimiento es procesado generando flujos dentro del sistema, para lo cual se definen unidades de conocimiento y se crean procesos y mecanismos de gestión que permitan incrementar y acelerar los procesos de aprendizaje; todo ello con vistas a lograr que las organizaciones sean más competitivas y adaptables a las nuevas complejidades; "la meta de la GC es mejorar la potencialidad de la creación de valores en la organización, mediante el uso más eficiente del conocimiento" (Edvinsson, 2001).

La GC se refiere a los asuntos críticos de adaptación y supervivencia de una organización, esencialmente, encarna los procesos organizativos que buscan la combinación sinérgica de datos, capacidad de procesamiento de información de tecnologías de la información, motivación y capacidad creativa e innovadora de los seres humanos. La GC se vincula con la creación y uso del conocimiento entre los miembros de una organización y su entorno, y a la vez dentro de la misma organización; permite abrir toda la riqueza del conocimiento existente, la cuestión clave es encontrar dónde están las oportunidades que permitan mejorar el rendimiento, y cuáles son las características del tipo de conocimiento que puede ser capturado y compartido; el capital intelectual de una organización es el activo más valioso que tiene, el conocimiento hace que los seres humanos creen a partir de él nuevas ideas, visiones e interpretaciones y lo apliquen en la toma de decisiones.

Nonaka (1999) la define como "aquellas acciones destinadas a organizar y estructurar los procesos, mecanismos e infraestructuras de la empresa con el fin de crear, almacenar y reutilizar los conocimientos organizativos. Puesto que la gestión de los conocimientos colectivos de una empresa guarda una estrecha relación con las diversas áreas funcionales de la organización, la gestión del conocimiento, debe partir del liderazgo de la alta dirección: es decir, un liderazgo que se extienda más allá de todo límite jerárquico o funcional,, definición 
en la que se puede observar el carácter dinámico de la función y su enfoque sistémico, primero parte de la idea de organizar los conocimientos (conjunto de ideas, de saberes, que han sido aprendidos gracias a determinado modelo mental), conocimientos que son las entradas del sistema que incorpora determinados procesos de transformación o de aprendizaje, gracias a los cuales se logrará crear nuevo conocimiento que le diferenciará del resto de sus competidores.

Según el modelo de Nonaka y Takeuchi (1999) (figura 3) una organización trabaja con tres clases de conocimiento: el conocimiento tácito, el conocimiento basado en normas, y el conocimiento de fondo. El conocimiento tácito se compone de las aptitudes, conocimientos técnicos especiales (know-how), heurística, intuiciones, y la satisfacción que la gente desarrolla a medida que se sumerge en el flujo de las actividades del trabajo, está profundamente arraigado en la acción y proviene de la participación simultánea de la mente y el cuerpo en la ejecución de tareas. Si bien el conocimiento tácito está implícito, el conocimiento basado en normas es conocimiento explícito que se utiliza para igualar las acciones a las situaciones mediante la invocación de normas adecuadas basadas en reglas que guían la acción; este tipo de conocimiento se utiliza en el diseño de las rutinas, procedimientos normalizados de trabajo y la estructura de registros de datos. El tercer tipo de conocimiento corresponde al de fondo, este es un conocimiento que es parte de la cultura organizacional, se comunica a través de textos orales y verbales tales como historias, metáforas, analogías, las visiones, las declaraciones de misión y el conocimiento previo que se basa en el marco cognitivo para la construcción de la realidad y la dota de sentido a las acciones de la organización y sus actividades.

FIGURA 3

Dimensión de la creación del conocimiento

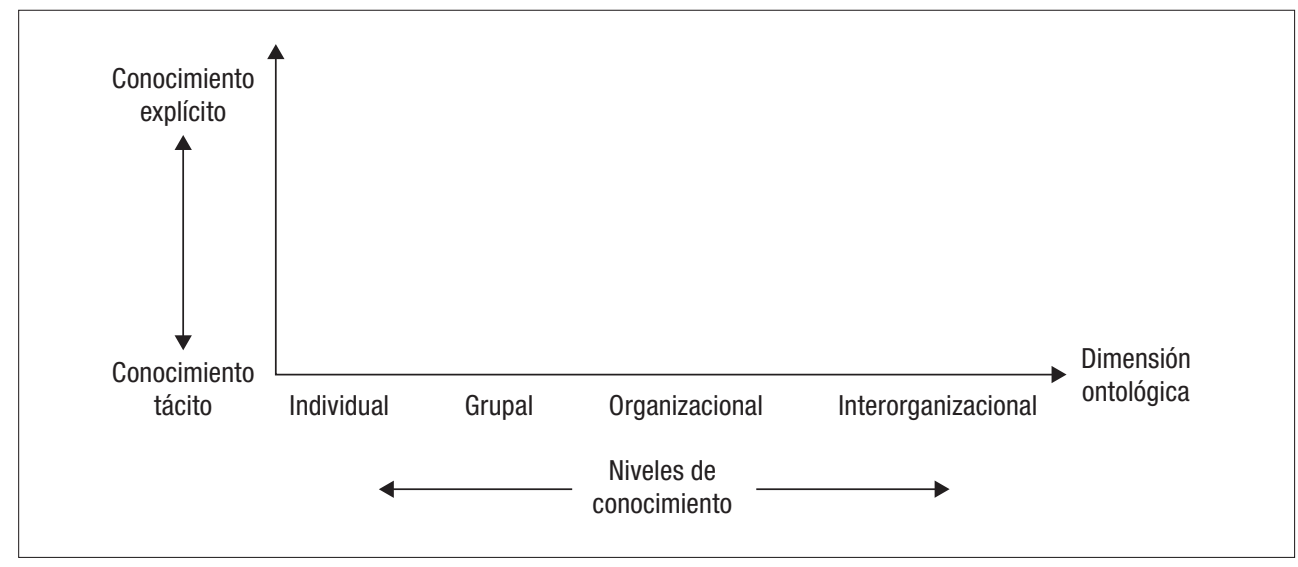

Fuente: Nonaka, I.; Takeuchi, H. (1999). 
El conocimiento explícito, es posible y conveniente almacenarlo en bases de datos, bases documentales como en intranets y extranets; pero no resulta factible estructurar y almacenar el conocimiento tácito, por lo que la mejor estrategia para gestionarlo es fomentar la creación de servicios y espacios que permitan el desarrollo de redes de colaboración entre las personas que componen la organización: el Chat, las listas de discusión, las comunidades virtuales, y otros, que se constituyen en fuentes importantes de conversión del conocimiento tácito en explícito, esto sucede con el registro de las acciones de la organización. En estas redes, pueden interactuar personas ajenas a la organización, siempre que sean personas con conocimientos sólidos e importantes para los objetivos a cumplir; a éstos se los convoca para que se incorporen a estas redes de colaboración, se produce entonces un proceso que se sustenta en el intercambio de información y conocimientos, donde al explicitarse estos últimos, se convierten en información que pueden utilizar otros; y a partir del registro de los datos e información sobre los conocimientos de cada persona, es posible la elaboración de mapas del conocimiento, en los que se especifique el saber de cada uno de los miembros de la organización.

La organización inteligente ha dominado una cuarta clase de conocimiento, de orden superior o meta-conocimiento, que se utiliza para crear, integrar y reforzar todos sus recursos intelectuales con el fin de alcanzar niveles superiores de rendimiento; Choo (1995) considera que el éxito de la GC conduce a una fase superior donde se generan las llamadas organizaciones inteligentes; a las que define como aquellas capaces de percibir y procesar información, crear conocimiento a partir de la información procesada y utilizar el conocimiento para la toma de decisiones de manera eficaz. En conclusión, la GC es una función dinámica o un concepto dinámico relacionado con la dirección o administración de un conjunto de flujos de conocimientos (externos e internos, captados o creados, explícitos o tácitos); el aprendizaje es el proceso de transformación y de incorporación del conocimiento tanto a nivel personal como grupal; y el capital intelectual es la medida del valor creado que permite explicar la eficacia del aprendizaje organizativo y evaluar, en suma, la eficiencia de la GC.

\section{Discusión y conclusiones}

Rastreando el origen y desarrollo teórico de los términos pueden observarse importantes diferencias y su distinción no parece ser un mero asunto semántico. La sociedad de la información sigue siendo el concepto dominante, considerada casi como un sinónimo de sociedad contemporánea, post industrial y definida porque en ella es clave la producción, distribución y manipulación de información; en cambio la conceptualización de sociedad del conocimiento trata de disociar la definición de la idea exclusiva de avances e innovaciones tecnológicas y de neto corte económico e incluir en su caracterización una dimensión de transformación global y pluralista del desarrollo basada en las capacidades cognitivas; si bien la sociedad de la información sólo constituye un objetivo o fin 
particular, el propósito más promovido es el establecimiento, a escala global, de la sociedad del conocimiento, como fuente de desarrollo para la humanidad, sin exclusión de países o personas.

Tres aspectos confluyen en las caracterizaciones que hemos desarrollado: información, personas y tecnologías de la información y la comunicación; la combinación de todas ellas da lugar a una serie de capacidades que son lo que constituye el capital de toda organización; tras reconocer su valor, las organizaciones deben trabajar para que los documentos, la información y el conocimiento que fluyen entre sus integrantes, no se pierda. Sin embargo el problema es el siguiente: si un alto porcentaje de documentos y contenidos de una organización se encuentran sin estructurar, las habilidades para utilizar esa información contenida en ellos decrecen considerablemente, para lo cual es necesario crear una infraestructura que integre la documentación estructurada con la no estructurada dentro de un verdadero sistema, de esta forma, el capital documental de una organización permite canalizar y agilizar los procesos informativos y la difusión de la información a través de los documentos, conformando un sistema de información. "Desde un enfoque meramente práctico, para conseguir una buena gestión de la información y el conocimiento, las empresas deben organizar y conseguir un buen sistema de gestión documental. Intentar establecer estrategias o programas de gestión del conocimiento sin tener esto resuelto, es como iniciar una reforma con la casa "sin barrer"; lo más probable es que el resultado más visible sea que "salga mucho polvo" (Bustelo Ruesta, 2000).

Incuestionablemente definir flujos de trabajo, especificando cómo se generan documentos y de dónde emanan, sitúa a la GD en el punto inicial de todo trabajo que aspire a desenvolverse dentro de este paradigma, que emplaza al archivo como un instrumento indispensable para la GI dentro de una organización, haciendo uso de tradicionales y nuevas formas documentales vinculadas a nuevas metodologías, procedimientos, y haciendo uso de las TIC. No obstante hay que tener en cuenta que nuestra profesión no cuenta con el monopolio del concepto GD, mientras nosotros nos quedamos con la elaboración de herramientas conceptuales como: clasificaciones, tesauros, procedimientos, aplicaciones concretas de las normas ISO, etc., los programadores informáticos utilizan este término para los softwares documentales y su mantenimiento.

El verdadero reto para las organizaciones consiste en transformar la documentación y la información en un recurso estratégico que facilite la toma de decisiones, dote de capacidad de reacción ante los cambios en el entorno, permita descubrir nuevas oportunidades de negocio y aumente la eficacia de la gestión empresarial; en definitiva, las organizaciones deben ser capaces de gestionar de manera sistemática e integrada, todos sus recursos de información, ya sea bajo la forma de información capturada del exterior o generada en el interior de la propia empresa. Y sobre todo, no hay que olvidar que el eje sobre el que gira la información son las personas, las que necesitan disponer de información para el correcto desempeño de sus funciones. Innegablemente las necesidades informativas variarán de acuerdo con las funciones y competencias que cada uno 
desempeñe, pero todo el personal es un potencial generador/usuario del sistema de información. Y sobre todo, en esto no existen demasiadas discrepancias, al menos teóricas; que si pueden existir en su implementación.

A nuestro entender el problema mayor reside en el establecimiento de diferencias entre los términos GI y GC, porque en la caracterización de los términos se observa que mientras el término GI se percibe como una actividad sin límites definidos, genérico, y sin rasgos conceptualmente diferenciados, la GC nace con términos que le son propios, tales como conocimiento explícito e implícito, que le otorgan fuerza a su desarrollo. Algunos autores como De Long y otros (1997) señalan que la GI se reduce a garantizar la accesibilidad y difusión de la información y la GC pone énfasis en añadir valor; Martesson (2000) argumenta que la GI basa su éxito en conservar y recuperar información, mientras la GC radica en el grado en que se comparta el conocimiento; Davenport y Prusak (2001) y Duffy (2001) explican las diferencias a partir del uso de la tecnología y plantean que mientras la GI se orienta a la gestión de objetos, la GC se orienta a encontrar, transmitir y conocer cómo se crean y utilizan los contenidos; Nonaka y Takeuchi (1999) plantean que GI se ocupa de gestionar la información y el conocimiento explícito, y que el conocimiento tácito es el objeto de la GC; Fahey (2001) define GC en torno a una enumeración de cuestiones que pueden ser resueltas a través de su implementación; Bustelo Ruesta y Amarilla Iglesias (2001) sostienen que la GC no arranca con la GI, sino que corresponde a un estadio superior; y Al-Hawamdeh (2002) identifica la GI como conocimiento explícito como información, y la GC como conocimiento tácito, difícil de convertir en un documento.

A la luz de la variedad de las apreciaciones vertidas en torno a los términos GI y GC se pueden identificar los siguientes aspectos: la GC se ubica indiscutiblemente centrada en un tipo particular de información: el Know How ${ }^{18}$ y emparentada con las Intranets; en el contexto de la GC se insiste en la trascendencia de la cultura corporativa para la integración y comunicación del conocimiento; y que existe un amplio nivel de subjetividad en la implantación de un proyecto de GC. "El fin último de la GC es permitir que el conocimiento sea compartido, para ser utilizado, y la mejor forma de hacer esto es exteriorizándolo, plasmándolo en un medio que lo haga accesible a sus usuarios. Esto es, creando documentos, pues la relación entre información y/o conocimiento en un soporte es igual al concepto documento. Luego también es clave en la GC la gestión documental” (Fernández Marcial, 2006).

Evidentemente, la GC implica crear valor a partir del capital intelectual, tiene como pilares a los recursos humanos, es una herramienta de gestión dirigida a aumentar tanto la productividad como la competitividad de la empresa mediante la creación de valor a partir de bienes intangibles; y se relaciona con la GI y la GD porque se sirve de todos los recursos de la organización, la creación de valor parte del conocimiento de clientes, trabajadores y colaboradores; supone recoger información, difundirla, sintetizarla, sacar conclusiones, definir métodos y fijar la cultura empresarial, lo cual permite localizar, organizar, transferir y hacer un uso eficiente de la información, que no sería posible si ésta se hallase frag- 
mentada; la GC crea valor a partir de la información (explícita y tácita) de la propia organización, el factor clave es el conocimiento de los trabajadores, y su objetivo es colocar a la empresa en una situación de liderazgo a partir del conocimiento, la creatividad, la capacidad de anticipación y la innovación.

Por esto la integración o punto común de las GD, GI y GC radica en el proceso de convertir el conocimiento tácito en explícito; Nonaka y Takeuchi (1999) expresan muy bien esta interrelación al establecer que el valor creado por una organización se encuentra determinado por la transferencia tácita y explícita de conocimientos entre individuos, y por la conversión del conocimiento de un tipo a otro; el tránsito de unos datos hacia la información y de ésta al conocimiento, es un proceso que consta de tres etapas, la primera representada por el "procesamiento de los datos", es decir por el uso de tecnologías que transforman aquéllos en información; la segunda etapa convierte ésta en conocimiento, gracias al "proceso de aprendizaje», es decir, de cómo saber "aprender a aprender» los conocimientos nuevos y, por último, la tercera etapa pretende que el conocimiento se transforme en "Competencia distintiva» o en otras palabras, en el soporte de la "capacidad de competir» o de "saber hacer» mejor que los demás, todo ello gracias a determinado "proceso de creación mental". En consecuencia este sistema lo que persigue es producir y difundir el saber, diseminar el talento humano o la inteligencia, tanto individual como organizativa por toda la estructura de la organización.

\section{FIGURA 4}

\section{Integración de los sistemas de gestión documental, de información $y$ del conocimiento}

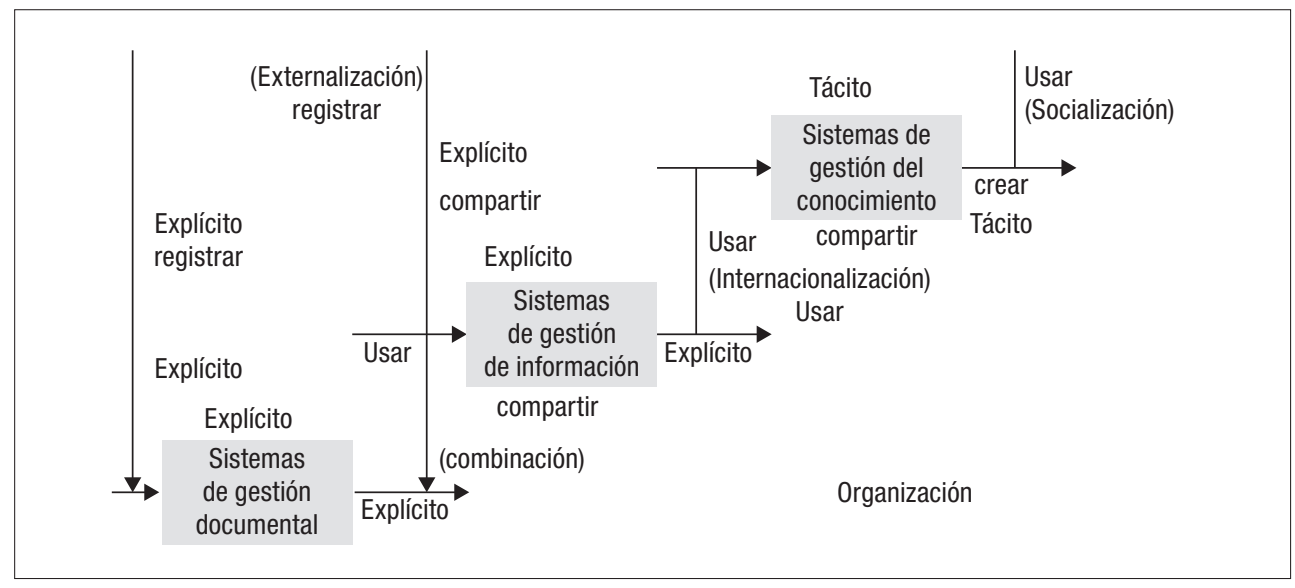

Fuente: Ponjuan Dante, Gloria (2006).

Finalmente, remarcar que el verdadero reto para las organizaciones es transformar sus documentos y la información en un recurso estratégico que les facilite la toma de decisiones, les dote de capacidad de reacción ante los cambios 
en el entorno, les permita descubrir nuevas oportunidades de negocio y que aumente la eficacia de la gestión empresarial.

Sin embargo, la gran antinomia consiste en que en la práctica la información disponible y el saber acumulado se han vuelto completamente inaccesibles para la mente humana que, en definitiva, se encuentra ceñida por sus limitaciones biológicas originales, y que ante la acumulación exponencial de información que nos inunda progresivamente, los seres humanos poseen la certeza de que resulta cada vez más difícil disponer de una visión equilibrada del conjunto; lo que genera como reacción una actitud de renuncia al conocimiento por desmotivación, por rendición, y una tendencia a aceptar de forma tácita la comodidad, eludiendo las argumentaciones racionales y las reflexiones intelectuales de cierta profundidad. La tecnología sobre la cual se mantiene la disposición del mundo en que nos hallamos, intensamente incomparable con el de hace algunas décadas, es de una complejidad y de un nivel de abstracción muy superior a la que sustentó la era industrial. "Es evidente que, a nivel profesional, el uso cotidiano como berramienta de trabajo de potentes ordenadores personales conectados permanentemente a una red global está modificando el ritmo y la secuencia de nuestros procesos mentales. Hoy es habitual manipular varios documentos a la vez mientras se recaba información en Internet, se atiende el correo electrónico o se mantienen conversaciones simultáneas a través de los servicios de mensajería instantánea. Ciertamente, desde un punto de vista productivo somos más eficientes, pero también se ha incrementado sensiblemente la complejidad de la mayoría de procesos, y el inmenso caudal de información que recibimos y que debemos gestionar amenaza con provocar nuevas formas de ansiedad. Es difícil focalizar $y$ centrarse, y esa necesidad de cambiar constantemente el foco de nuestra atención acaba por modelar nuestra forma de razonar hasta ubicarnos en un estado de dispersión que, conceptualmente, es incompatible con la concentración que requiere cualquier reflexión de cierta consistencia" (Brey, 2009).

Esto recién comienza y queda mucho camino por recorrer, ¿los profesionales de la información seremos capaces de llegar al centro de los procesos informacionales en las organizaciones, para entregar soluciones productivas y eficientes a través de sistemas de información?

\section{Notas}

[1] Término acuñado por Alfons Cornella para referirse a la fascinación por la información excesiva.

[2] El término puede ser tanto una unidad léxica simple, como la combinación de varias unidades; el término designa los objetos de la realidad y la naturaleza lógica de los objetos de conocimiento o términos, incluyendo los nombres de propiedades (adjetivos), acciones (verbos) e incluso relaciones; por tanto, aunque se suele afirmar que el sustantivo es la unidad terminológica por excelencia, no hay que pasar por alto que otras unidades pueden ser igualmente características de una terminología de un dominio concreto. 
[3] El término relación es entendido como la acción y efecto de referir, establecer conexiones y correspondencias de un término con otro; cualquier tipo de conexión existente entre los elementos tanto desde el punto de vista intencional (proposicional diádica) como desde el punto de vista extensional caracterizando las diferencias de sus elementos debidas al orden.

[4] Si bien el término gestión posee su equivalente Management en lengua inglesa, en ocasiones este término ha sido desacertadamente traducido al castellano como administración.

[5] El ciclo de vida de un documento es un concepto clásico de la archivística moderna que se refiere a las distintas fases o etapas por las que pasan los documentos, y a las transformaciones en sus valores primarios, desde su creación, como producto de la actividad humana, hasta a su eliminación o selección para su custodia permanente, generalmente por su valor secundarios (histórico).

[6] Aquel que va unido a la finalidad inmediata por la cual el documento se ha producido por la institución de carácter fiscal, judicial, jurídico-administrativo, etc. (Diccionario de Terminología Archivística, 1993).

[7] Aquel que posee del documento como fuente primaria para la historia. (Diccionario de Terminología Archivística, 1993).

[8] En 1972 Wiffels planteó la teoría de las tres edades, que se constituyó en la piedra angular de la gestión documental y base conceptual de la operativa de los programas de gestión documental en las instituciones. Esta teoría define tres edades o etapas del documento que, a su vez, diferencian tres tipos de archivo determinados por la consulta y trámite de los mismos.

[9] El canadiense Michel Roberge es presidente de Solutions Documentaires GESTAR y uno de los mayores especialistas mundiales en gestión documental.

[10] El término inglés "records" designa los documentos reconocidos como evidencia y reflejo de las actividades de la institución que los ha creado (documentos de archivo), a diferencia de los términos "documents", que sólo tiene en cuenta su contenido informativo, y "archives", que tiene un sentido histórico.

[11] En el mundo anglosajón los sistemas de gestión documental se identifican con las siglas: DMS (Document Management System) o RMS (Records Management System).

[12] Según los estudios de Gartner Group, entre un 10 y un $20 \%$ del tiempo de cualquier trabajador.

[13] Alfresco (http://www.alfresco.com); Apache Jackrabbit (http://jackrabbit.apache.org); DocMGR (http://www.docmgr.org/); Docuges (http://docuges.sourceforge.net/); Knowledge tree (http://www.knowledgetree.com/opensource); Maarch (http://www. maarch.org); MyDMS (http://sourceforge.net/projects/mydms/); Nuxeo 5 (http://www. nuxeo.com); OpenKM (http://www.openkm.com); Quotero (http://www.quotero. com).

[14] Para profundizar en la información sobre los casos Enron y Arthur Andersen se puede consultar: Mattessich, Richard. Lecciones de Enron y Arthur Andersen Co. Disponible en: <www.uam.es/personal_pdi/economicas/lcanibano/2007/Tema\%202\%20 Gestion\%20de\%20beneficios/Enron-Malaga_LV.pdf>

[15] Basada en la norma australiana AS 4390. Australia ha sido el primer país en desarrollar un estándar para la gestión de los «records». 
[16] La teoría matemática de la información, desarrollada inicialmente, en 1948, por el ingeniero electrónico estadounidense Claude E. Shannon, en su artículo, "A mathematical Theory of Communication", se ocupa de la medición de la información, de la representación de la misma y de la capacidad de los sistemas de comunicación para transmitir y procesar información.

[17] La información puede entenderse como un dato dotado de significado, como una proposición, pensamiento o idea susceptible se ser verdadera o falsa.

[18] Know-How (saber-cómo) expresión anglosajona que refiere a conocimiento fundamental y a una forma de transferencia de tecnología.

\section{Bibliografía}

Al-Hawamdeh, S. (2002): Knowledge Management: re-thinking information management and facing the challenge of managing tacit knowledge. Information Research, 8 (1): 1-39. Disponible en: <http://informationr.net/ir/8-1/paper143> [Consulta: 11 de noviembre de 2011].

Alòs Moner, A. d' (2011): Gestión de documentos: sonrisas y lágrimas. Anuario ThinkEPI, (5). Disponible en: <http://www.thinkepi.net/gestion-de-documentos-sonrisas-y-lagrimas> [Consulta: 11 de noviembre de 2011].

Belkin, N. J. (1978): Progress in Documentation: information concepts for Information Science. Journal of Documentation, 34 (1): 55-85.

Bell, D. (2001): El advenimiento de la sociedad post-industrial: un intento de pronosis social. Madrid. Alianza. (Original norteamericano 1973). Disponible en <http://www. infonomia.com/articulo/libros/6151> [Consulta: 11 de noviembre de 2011].

Brey, A.; Innerariti, S.; Mayos, Gonçal (2009): La sociedad de la ignorancia: y otros ensayos. Barcelona; Infonmía.

Brookes, B. C. (1980): The foundation of Information Science. Journal of Information Science (2): 125-33.

Bustelo Ruesta, C. (2000): Gestión documental en las empresas: una aproximación práctica. VII Jornadas Españolas de Documentación: FESABID. Bilbao, 19-21 de octubre. Disponible en: <http://www.delfos.co.cu/boletines/bsa/content_gest_docm\%2010. html> [Consulta: 11 de noviembre de 2011].

Bustelo Ruesta, C.; Amarilla Iglesias, R. (2001): Gestión del conocimiento y Gestión de la información. Boletín del Instituto Andaluz de Patrimonio Histórico, VIII (34): 226-230.

Bustelo Ruesta, C. (2011a): Los grandes temas relacionados con la gestión de documentos: desafíos y oportunidades. El profesional de la información; 20 (mar-abr): 129- 133. Disponible en: <http://www.elprofesionaldelainformacion.com/contenidos/2011/marzo/01.pdf> [Consulta: 11 de noviembre de 2011].

Bustelo Ruesta, C. (2011b): Serie ISO 30300: sistema de gestión para los documentos. Madrid: SEDIC: disponible en: <http://www.sedic.es/formulario-descarga-DT2.asp> [Consulta: 11 de noviembre de 2011].

Choo, C. W. (1995): Information management for the intelligent organization: roles and implications for the information professions [documento electrónico]. Digital Libraries Conference, Singapur; Mar 27-28. Disponible en: <http://choo.fis.utoronto.ca/fis/respub/dlc95.html> [Consulta: 3 de junio de 2011]. 
Cornella, A. (2009): Infoxicación: buscando un orden en la información. Barcelona: Infonomía.

Davenport, T.; Prusack, L. (2001): Conocimiento en acción: cómo las organizaciones manejan lo que saben. México: Prentice Hall.

De Long, D.; Davenport. T.; Beers, M. (1997): Working knowledge: how organizations manage what they know. Boston: Harvard Bussiness Press.

Drucker, P. F. (1969): La era de la discontinuidad. New York: Harper \& Row.

Drucker, P. F. (1994): La sociedad postcapitalista. Buenos Aires: Sudamericana.

Duffy, J. (2001): The tools and technologies needed for knowledge management. Information Managment Journal, 35 (1): 64-67.

Edvinsson, L. (2001): Developing Intellectual Capital at Skandia: long Range Planning. En: Mac Morrow, N. Knowledge management an introduction. Annual Review of Information Science and Technology, vol. 35, pp. 381-422.

Fahey, L.; Srivastava, R.; Sharon, J. S.; Smith, D. E. (2001): Linking e-business and operating processes: the role of knowledge management. IBM Systems Journal, 40 (4): 889-907.

Fernández Marcial, V. (2006): Gestión del conocimiento versus Gestión de la información. Investigación Bibliotecológica, 20 (41): 44-62.

Llansó i Sanjuan, J. (1993): Gestión de documentos: definición y análisis de modelos. Euskadi: Irargi.

Lodolini, E. (1993): Archivística: principios y problemas. Madrid; Asociación Española de Archiveros, Bibliotecarios, Museólogos y Documentalistas.

López Hernández, J. (1990): La Gestión de la información en las organizaciones: una disciplina emergente. Revista general de información y documentación, 1 (2): 7-22. Disponible en: <http://www.ucm.es/BUCM/revistas/inf/02104210/articulos/DCIN9090110133A.pdf> [Consulta: 6 de junio de 2011].

Machlup, F. (1962): The production and distribution of knowledge in the United States. Princeton, N. J.: Princeton University Press.

Malhotra, Y. (2000): Knowledge management and virtual organizations. Hershey (USA), London: Idea Group Publishing. Disponible en: <http://books.google.com/books?id=

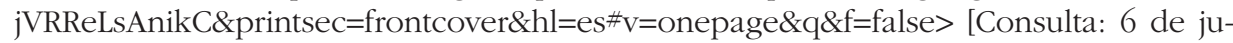
nio de 2011].

Martesson, M. (2000): A critical review of knowledge management as management tool. Journal of Knowledge Management, 40 (4): 204-216.

Martínez Méndez, J. (1995): El salto desde la gestión de información a la gestión del conocimiento. Scire: 5 (1). Disponible en: <www.um.es/gtiweb/fjmm/elsalto.doc $>$ [Consulta: 6 de noviembre de 2011].

Mansell, R.; W. U. (eds.) (1998): Knowledge Societies: information technology for sustainable development. Oxford; Oxford University Press. Disponible en: <http://www.sussex. ac.uk/spru/research/ink/knowledgesocieties> [Consulta: 19 de abril de 2011].

Masuda, Y. (1984): La Sociedad de la Información como sociedad postindustrial. Madrid: Tecnos.

Moro Cabero, M. (2011): Certificación de calidad en los archivos: análisis y prospectiva. Revista Española de Documentación Científica, 34 (3): 447-460. 
Mount, E. (1966): Information Management in Engineering Education: proceedings and recommendations of the Conference on Information Sources, Systems and Media in Engineering Education. American Documentation, 18 (2): 116-117.

Nonaka, I. (1999): La organización creadora de conocimiento. México: Oxford.

Nonaka, I.; Takeuchi, H. (1999): La organización creadora del conocimiento: cómo las compañias japonesas crean la dinámica de la innovación. México DF: Oxford University Press.

Norma ISO UNE 15489 (2006): Información y documentación: gestión de documentos. Ginebra: ISO

Ponjuan Dante, G. (2004): Gestión de información: dimensiones e implementación para el éxito organizacional. Rosario (Argentina): Nuevo Paradigma.

Roberge, M. (2006): Lo esencial de la gestión documental. Quebec: Gestar.

Schellemberg, T. R. (1956): Modern archives: principles and techniques. Chicago; University of Chicago Press. Disponible en: <http://www.archivists.org/publications/epubs/ ModernArchives-Schellenberg.pdf> [Consulta: 6 de junio de 2011].

Shannon, C. E. (1949): The mathematical theory of communication. Illinois: Univesity Illinois Press.

Stehr, N. (1984): Society and Knowledge: contemporary perspectives in the Sociology of Knowledge. London: Transaction Books.

Unesco (2005): Hacia las sociedades del conocimiento. París: UNESCO. Disponible en: $<$ http://unesdoc.unesco.org/images/0014/001419/141908s.pdf> [Consulta: 6 de junio de 2011].

White, M. (1985): Intellingence management. En: Cronin, Blaise (ed.). Information Management: from strategies to action. London: ASLIB, pp. 19-35.

Woodman, L. (1985): Information managment in large organizations. EN: Cronin, Blaise (ed.). Information Managment from strategies to action. London: ASLIB, pp. 95-114. 